\title{
Savoirs juridiques et savoirs sur le droit
}

Brève intervention à propos du texte introductif de Denise Pumain

Pierre Moor

\section{OpenEdition}

Journals

Édition électronique

URL : http://journals.openedition.org/ress/362

DOI : $10.4000 /$ ress.362

ISSN : 1663-4446

Éditeur

Librairie Droz

Édition imprimée

Date de publication : 1 février 2005

Pagination : 13-22

ISBN : 2-600-00958-2

ISSN : 0048-8046

Référence électronique

Pierre Moor, "Savoirs juridiques et savoirs sur le droit », Revue européenne des sciences sociales [En

ligne], XLIII-131 | 2005, mis en ligne le 04 novembre 2009, consulté le 30 avril 2019. URL : http://

journals.openedition.org/ress/362; DOI : 10.4000/ress.362 


\section{Pierre MOOR}

\section{SAVOIRS JURIDIQUES ET SAVOIRS SUR LE DROIT Brève intervention à propos du texte introductif de Denise Pumain}

\section{INTRODUCTION : LE DROIT COMME SCIENCE?*}

«Comment les savoirs produits par les sciences sociales peuvent-ils s'accumuler, se cumuler, au cours du temps dans une discipline, et entre les différentes disciplines?»

Je me suis arrêté à la phrase liminaire du texte introductif de Denise Pumain ${ }^{1}$ parce que, tout d'un coup, il m'est venu à l'esprit que le statut du droit dans cette assemblée n'avait jamais été mis en question, alors même que, appliqués à cette discipline précise, les mots employés font à ce point problème qu'il est impossible de continuer sans le résoudre.

En d'autres mots : le droit est-il une «science»? Produit-il des «savoirs »? Estil une discipline des sciences sociales au même titre que la sociologie, l'économie, la politologie, la géographie, la philosophie? Avons-nous, nous juristes, le droit de nous asseoir à la même table que vous autres? Quel discours juridique pouvonsnous tenir que vous puissiez entendre pour l'intégrer dans vos propres disciplines et quel discours sociologique, économique, etc., pouvez-vous tenir pour que nous puissions l'intégrer dans notre savoir de juriste?

L'activité scientifique a pour visée d'introduire la plus grande rationalité possible dans la connaissance d'un objet: c'est une définition dont je ne parierai pas qu'elle est philosophiquement orthodoxe, mais qui devra suffire ici. Les concepts, les théories, les définitions et leurs interprétations (pour reprendre les divers termes que Denise Pumain emploie dans la seconde phrase de son texte, et qui paraissent, pour elle, caractériser ce qu'est une «science» et ses «savoirs») sont la manifestation intellectuelle de cette rationalité en tant qu'elle décrit un objet qui, d'une manière ou d'une autre, lui est extérieur, dans une autonomie d'existence dont précisément il s'agit de rendre compte.

Voyons ce qu'il en est de ce point de vue de la scientificité du droit. Cela implique que l'on distingue dans le phénomène dit droit plusieurs niveaux. Le premier est celui de l'activité juridique proprement dite - niveau positif : la signification donnée à un rapport entre deux ou plusieurs personnes, en fonction d'un ensemble déterminé de textes de référence (que j'appelle des textes normatifs), signification qui, selon l'ordre juridique qui comprend ces textes, devient à un certain moment incontestable. Le deuxième niveau est celui de la théorie du droit:

* Nous avons conservé le style oral de notre participation au colloque.

Dans ce volume, p. 5. 
à savoir l'élaboration des concepts nécessaires pour décrire le fonctionnement de l'ordre juridique comme tel - niveau théorique ${ }^{2}$. La philosophie du droit a une position ambiguë: d'une part théorie du droit considéré pour lui-même, d'autre part intégration du droit dans un système philosophique global. Il y a certes encore d'autres approches possibles, mais, comme elles prennent le droit comme objet et sont ainsi incontestablement des sciences au sens que j'ai indiqué plus haut, elles sont hors de mon propos - principalement la sociologie du droit.

Je vous entretiendrai ici d'abord du premier niveau, dans un premier chapitre; Gérard Timsit traitera du deuxième. Dans mon second chapitre, j'essaierai de montrer cependant un emprunt que la théorie du droit a fait à une discipline philosophique et la leçon que, peut-être, on pourrait en tirer.

\section{LE NIVEAU DU DROIT POSITIF}

1.1. Le premier niveau, le plus manifeste et le plus quotidien, celui de l'activité juridique elle-même, est celui où le droit s'applique: je l'appellerai le niveau positif. Par son travail sur des textes normatifs à partir desquels il construit une argumentation, le juge définit juridiquement une certaine situation et lui attribue en conséquence un statut de droit. Activité certes non déductive, mais visant néanmoins la plus grande rationalité possible, le droit comme discipline produit ainsi des «connaissances», qu'il répétera en les reproduisant dans tous les cas analogues.

En comparaison avec celles des sciences, ces «connaissances », me paraît-il, se présentent de façon radicalement différente, parce qu'elles se confondent avec leur objet, et même plus: elles sont leur objet. Il n'y a pas, d'une part, l'objet «contrat de leasing », ou l'objet « recours pour excès de pouvoir», et, d'autre part, un savoir, ou une définition du contrat de leasing, du recours pour excès de pouvoir. La définition juridique du contrat, du recours est l'objet juridique dit «contrat de leasing», « recours pour excès de pouvoir». La «science juridique»on s'exprime parfois ainsi - est ainsi même plus que la connaissance de son propre contenu, elle est son propre contenu: le savoir juridique est le droit lui-même. La locution est bien connue: le juge dit le droit, et, le disant, le fait.

Développons un autre exemple. En principe, les libertés publiques sont des garanties offertes par l'ordre juridique aux administrés contre les actes de puissance publique; elles ne valent pas dans les relations entre particuliers. Ce principe est-il un concept, une théorie, une définition? C'est une définition, celle de la portée des libertés publiques, définition qui est celle de la pratique juridique ellemême, et non pas un savoir sur cette pratique. Par conséquent, en application de cette définition, un employé du secteur privé qui se plaindrait en justice de ce que son employeur ne respecte pas sa liberté d'expression se verrait éconduit. À moins qu'une nouvelle pratique ne soit instaurée - une nouvelle définition, adoptée par l'organe constituant ou résultant d'un revirement de jurisprudence dans l'inter-

2 La description donnée du niveau positif est faite dans des termes empruntés au niveau théorique. Cf., sur cette description, Pierre Moor, L' 'euvre du droit, Paris, PUF, à paraître (2004/5), chap. VII et VIII. 
prétation de la garantie; et cette définition ne se serait pas un nouveau savoir sur la portée de la liberté publique, comme si celle-ci était un objet préexistant dont on comprendrait mieux la rationalité.

Quel est alors le statut de la définition? Elle est l'élaboration de la pratique en termes généraux et abstraits - soit que, comme texte légal ou constitutionnel, elle la précède, soit que, comme maxime qui la résume, elle l'accompagne. Elle est une mise en forme de la pratique à laquelle celle-ci se réfère pour se définir ellemême. La «science juridique » est bien la connaissance de ces définitions en tant qu'elles ont été et continuent d'être pratiquées, de façon que, dans un cas concret nouveau, elles soient à nouveau appliquées de manière cohérente; mais, en même temps, c'est elle qui élabore ces définitions. Elle le fait en interprétant le texte légal - et cette interprétation est le sens même de ce texte - ou en ramassant sous une forme synthétique un ensemble de décisions judiciaires - et c'est, au sens matériel du terme, la jurisprudence, sous l'aspect de sa continuité. La science, la pratique de cette science et son objet se confondent.

1.2. Cette confusion est loin d'être une tare, tout au contraire. Elle définit le droit, dans son fonctionnement propre, par l'autonomie qu'elle lui confère par rapport à tout pouvoir extérieur (c'est-à-dire non juridique). Le développement du savoir juridique a lui-même pour objet; le droit se déploie lui-même en se connaissant. Il est, sous cet angle, une activité de production de sens qui se caractérise par l'autoréférentialité des connaissances qu'elle produit. Dans quelles conditions cette activité se produit, quelles différenciations internes elle implique, tout cela mériterait une explicitation qui relève de la théorie du droit. Quant à l'analyse des rapports de cette autonomie avec l'environnement politique, économique, social, elle appartient à la sociologie et à la politologie; et celle de son statut - fictif ou factuel -, je dirais qu'elle incombe à la philosophie du droit.

Au niveau positif donc, l'accumulation des «savoirs » est consubstantielle au droit: c'est elle qui assure la permanence du droit, dans une continuité du discours cependant qui ne peut ignorer la nécessité d'évoluer - mais à l'intérieur de son propre système. Et cela précisément parce que, face à l'imprévisibilité des situations extérieures auxquelles elle est confrontée, la «science juridique » n'est en droit de les traiter que par rapport à ce qu'elle est déjà. L'ordre juridique, en effet, ne peut se concevoir que comme autoréférentiel, parce qu'il serait autrement impossible aux acteurs sociaux de s'y référer pour anticiper les conséquences de leurs actes. La fonction propre de la «science juridique» est donc bien de se dire elle-même, en se reconnaissant elle-même dans la définition qu'elle se donne des situations extérieures qui sont soumises à son jugement.

Si le «savoir juridique» est connaissance de soi-même, cela implique une structure particulière du phénomène de l'accumulation et de l'innovation. Il y a bien possibilité d'innovation, mais par redéploiement, renouvellement, reconnaissance de ce qui est déjà su. L'accumulation est intégrative; ou, vu du point de vue inverse, l'intégration est cumulative.

La réponse que l'ordre juridique doit donner à une question nouvelle que son environnement lui pose doit obéir à une double exigence: premièrement, être adéquate à la situation de fait qui provoque cette question et, secondement, être intégrée dans cet ordre juridique tel qu'il est constitué. Le droit a ainsi une position paradoxale: sa rationalité est autonome, en ce sens qu'il doit trouver en lui- 
même sa propre vérité, mais sa finalité est hétéronome, puisque la solution vraie qu'il élabore par son travail sur le savoir qu'il a de lui-même est destinée à régler une question dont les enjeux lui sont extérieurs.

1.3. Précisons ce que nous avons appelé la «science juridique»: nous entendons par là toute argumentation quelconque dont le but est de justifier le sens juridique d'une relation entre sujets de droit. Sous cet angle, nous ne faisons aucune différence entre les considérants d'une sentence judiciaire, la motivation d'un mémoire déposé en justice par un avocat, l'avis de droit rédigé par un professeur. Il y a certes une différence, mais elle réside dans l'autorité attachée au dispositif du jugement (la «force de chose jugée»), et non pas dans l'apport de connaissance que celui-ci fournirait à la «science juridique». Argumentativement, le jugement est structuré comme le mémoire de l'avocat ou l'avis de droit d'un expert: il fait appel aux mêmes textes et aux mêmes figures de raisonnement; sa «science» est la même. Seule différence: l'autorité attachée à sa conclusion, mais celle-ci ne concerne plus la scientificité de l'acte.

L'auteur du mémoire de recours ou de l'avis de droit peut donc bien faire erreur, en ce sens que le discours qu'il tient est infirmé par le juge. Mais cette erreur se révèle a posteriori - même plus : elle n'existe qu' a posteriori. Car elle ne découle pas d'une irrationalité intrinsèque au discours tenu: elle provient de ce que le juge a en monopole la compétence d'un dire définitif sur ce qui est vrai, ce qui ne préjuge en rien de la scientificité de ce dire.

On objectera qu'il existe bien des erreurs a priori: lorsque la solution découle clairement et sans ambiguiité aucune d'un texte légal - ainsi lorsque la loi fixe un délai à dix jours, et que l'avocat dit à son client qu'il est de deux semaines. L'avocat a un savoir erroné. On notera toutefois que ce genre d'erreur peut aussi arriver à un juge. A-t-elle alors le même statut que celle de l'avocat? Apparemment non: car il n'existe personne qui, dans l'ordre juridique, puisse la constater de manière incontestable. Certes, la contradiction entre le texte de loi et l'arrêt peut être manifeste: mais personne d'autre que le juge n'a la compétence de le dire, ce qui signifie que chacun doit tenir l'erreur judiciaire pour vraie jusqu'à ce que, dans un cas ultérieur (dans une autre cause) ou un arrêt ultérieur (dans la même cause, sur recours ou révision) et sans effet sur la cause précédente, un autre juge statue.

Il en va de même des controverses doctrinales. Elles portent non pas un savoir sur ce qui est, mais sont elles aussi des anticipations ${ }^{3}$ en ce sens que chacune d'elles propose sa solution comme devant être considérée en tant qu'étant vraie. Le passage de ce devoir être à l'être se réalisera dans la parole du juge.

La vérité est par conséquent dans le dire du juge. Et l'erreur que commet un avocat est une erreur sur ce que le juge va dire - une fausse anticipation juridique, et non pas un savoir erroné. La vérité: le concept mérite d'être explicité. Elle relève en même temps de l'absolu et du relatif. De l'absolu, parce qu'est vrai ce que dit le juge, dans l'acte même du dire, et cela de manière incontestable dès que l'arrêt est définitif. Mais la solution doit être argumentée, et c'est ce qui fait la spécificité du droit comme organisation de pouvoir. La vérité en tant qu'argu-

3 Sur ce concept, cf. Moor, op. cit., chap. VII. 
mentée, comme motivation (et non comme dispositif du jugement) entre dans les circuits de communication constituant l'univers juridique, et peut être contestée dans sa rationalité: elle est en ce sens donc aussi relative, mais restera vraie malgré les critiques - jusqu'à ce que le texte de référence (la loi qui lui sert de fondement) soit modifié ou, par revirement de jurisprudence, fasse l'objet d'une autre interprétation - nouveau savoir dans une nouvelle parole.

De nouveau, dans l'institution judiciaire, on débouche sur le concept de compétence; et on remarque que le terme a un autre sens que dans le langage courant. Ce dernier - qui est d'ailleurs postérieur au sens juridique - sert à désigner une personne «qui a l'aptitude voulue pour décider de quelque chose, qui connaît bien une question», et a pour synonyme «capable, qualifié »; il s'agit d'évaluer son savoir, ses connaissances ${ }^{4}$. L'autorité compétente - au sens juridique - est celle qui a le pouvoir de décision. On dira d'ailleurs volontiers les compétences au sens courant, mais la compétence au sens juridique; et on peut remarquer que le terme de connaissance s'emploie de même au singulier dans le jargon juridique (dans un sens proche de compétence) et souvent au pluriel dans le sens courant (comme ci-dessus, par exemple): le juge connaît de telles catégories de causes.

Cette compétence, dans laquelle se confondent le dire et le savoir, serait pur pouvoir si elle n'était pas médiatisée par l'obligation de se référer à des textes dont la formulation (mais non la signification) échappe à son emprise. Le dire est un savoir sur ces textes; il se manifeste dans un discours argumentatif qui amène ces textes à la parole (pour reprendre une expression de Paul Ricœur ${ }^{5}$ ).

1.4. Cela étant, plusieurs questions sont ainsi soulevées:

— comment se fait l'accumulation des savoirs et des «paroles»?

— comment cette accumulation, et dans quelle mémoire, intègre-t-elle les évolutions?

— comment se font les ruptures internes au système (les changements de pratique, les revirements de jurisprudence) et celles qui sont externes (la modification des textes normatifs de référence) (si tant est d'ailleurs qu'il y ait une différence entre «interne» et «externe»)?

Nous rentrons là dans le domaine de la théorie du droit, que nous n'aborderons pas ici, pour renvoyer à l'intervention de Gérard Timsit.

1.5. Mais auparavant, il importe de souligner que la description que j'ai faite n'aurait pas été possible sans un emprunt à une autre discipline, dont ma référence à Paul Ricœur vous aura fait deviner l'identité. Si on comprend dans la philosophie du droit l'analyse de son mode propre de rationalité (son épistémologie), et non seulement celles de son fondement (son ontologie) et de la mesure de sa valeur (son éthique), j'ai pénétré, par le fait même d'expliquer ce qu'était le savoir juridique, dans le jardin du philosophe. Et, sans que je l'aie explicité, le concept

4 Sic, Lexis, Paris, Larousse, 1975.

5 Citation déjà faite dans Moor, op. cit., chap. VII. 
qui m'a servi de viatique est celui de texte, dans l'acception qu'en a élaboré l'herméneutique et que je lui ai empruntée. Je peux ainsi entreprendre la deuxième partie de l'exposé, qui sera l'analyse que je peux faire à la lumière de l'exemple de cet emprunt.

\section{QU'EST-CE QU'ON EMPRUNTE?}

2.1. Sous quelle forme s'exportent et s'importent les concepts élaborés dans une discipline? Ce qui est d'abord apparent, c'est que le nom reste identique; mais on ne peut rien en déduire. Restent-ils tels quels, c'est-à-dire un concept identique? Ou l'opération les transporte-t-elle comme une simple image, servant provisoirement de métaphore permettant de figurer un concept qui, pour servir dans la discipline de réception, doit encore être élaboré? Ou, enfin, le transfert n'est-il que celui du nom?

Je ne connais pas, malheureusement, d'exemple d'exportation de concepts juridiques - sauf, peut-être, celui de «forclusion »: juridiquement, est forclos celui qui est déchu de son droit d'agir en justice pour avoir laissé passé le délai dans lequel il devait agir. Quel est le sens de «forclusion» dans la théorie psychanalytique? Je soupçonne un usage métaphorique.

Je connais en revanche des termes que l'on retrouve dans diverses disciplines sans que je puisse dire qu'il y en a un concept unique, ni même s'il y a une simple analogie de sens; tout ce que je sais, c'est qu'ils proviennent de quelque part, mais peut-être ont-ils de multiples origines, ou datent-ils d'une période dans laquelle la science se mettait au singulier. Nous nous sommes arrêtés il y a dix ans, dans le premier colloque de notre groupe ${ }^{6}$, sur le terme de loi, mais je ne me souviens pas que nous soyons arrivés à une quelconque conclusion. Un autre terme communément utilisé est celui de système. Ces deux cas présentent un degré d'abstraction élevé. Il n'en va pas toujours ainsi; figure plus concrète, se répand aujourd'hui, par exemple, le mot réseau: «ensemble de lignes entrelacées, de points communiquant entre eux », dit le dictionnaire. Donc une métaphore, dont il est même difficile de savoir si elle n'a pas pour seul effet de montrer que les choses sont plus compliquées qu'on a bien voulu le croire - ce qui en ferait, moins qu'une métaphore, une pure image.

Un ouvrage de théorie juridique récent s'intitule ainsi «De la pyramide au réseau? Pour une théorie dialectique du droit ${ }^{7}$. Dialectique ne me paraît d'ailleurs pas pris au sens hégélien, mais semble signifier, pour les auteurs de ce livre, communication discursive dans un système où les hiérarchies sont flottantes ou, en termes moins imagés, «complexes, relatives et récursives». Réseau n'est ici qu'une image, et les trois adjectifs par lesquels la nouvelle conception veut se caractériser concluent d'ailleurs abstraitement la description d'une lithographie où l'on voit un ensemble de lignes qui s'entrecroisent en formant un certain

- Cf. De la logique des lois, Ier séminaire interdisciplinaire du Groupe d'études «Raison et rationalités », Actes édités par Marie-Jeanne Borel et Pierre Moor, Revue européenne des sciences sociales 34 (1996), Nº 104.

7 François m/Michel van de Kerchove, De la pyramide au réseau? Pour une théorie dialectique du droit, Bruxelles, Facultés universitaires Saint-Louis, 2002. 
nombre de pyramides. Ce qui fait douter de son utilisation comme concept, c'est que l'image ne rend pas compte du mouvement qui anime le tout, et elle n'est donc même pas une métaphore adéquate de la dialectique du système (cette fois au sens logique du terme).

Je crains qu'il n'en aille de même pour beaucoup de ces apparents transferts. L'habit ne fait pas le moine. Il y a parfois de simples homographies, au lieu de l'homologie postulée. Je soupçonne que loi n'est pas loin d'en être un bon exemple: concept unique, analogie, image, homographie?

2.2. Le cas de loi offre d'ailleurs un bon exemple des effets pervers que peut avoir la circulation à travers différentes disciplines d'un terme qui sert de concept. Prenons la méthodologie juridique positiviste, qui a régné longtemps et qui imprègne encore sans doute l'appréhension commune de ce que devrait être le droit dans un Etat de droit: elle repose sur l'idée que la loi, dans sa formulation générale et abstraite, contient par avance et exhaustivement tout concret, dont la détermination se fait par une opération simple de déduction - le fameux syllogisme juridique. On peut être certain d'une part que cette idée est fausse, parce qu'elle ne rend aucunement compte de la réalité de l'activité juridique. On peut d'autre part avancer l'hypothèse que cette idée de la loi a pu dominer la théorie du droit parce qu'elle s'est mise au bénéfice de l'idéal de rationalité représenté par le concept de loi qui s'est élaboré auparavant dans les sciences exactes.

Il faut donc savoir ce que l'on importe: un concept ou une théorie, soit un ensemble de concepts impliqués les uns aux autres - théorie signifiant (aussi!) «longue suite d'êtres animés qui marchent l'un derrière l'autre ${ }^{8}-$ et pourquoi pas une longue suite de concepts! Peut-on prendre le concept sans la suite? $\mathrm{Ou}$ bien l'être (en l'occurrence intellectuel) perd-il son sens si on le sort de la suite? La même question s'illustre avec l'image plus à la mode du réseau: tout concept est le point où s'entrelacent les lignes d'un réseau, et il a valeur explicative en ce point précis. Peut-on le découper pour le greffer ailleurs? La réponse devrait être négative, si la métaphore est adéquate: si le «réseau» est un concept, il est concept uniquement parce que intégré dans un réseau.

Ou bien il faudrait différencier: ce n'est pas le point que l'on découpe, mais l'entrelacement; on ne prend pas le concept, mais l'ensemble de la théorie qu'il implique; et c'est là qu'il faut faire attention. L'exemple de la loi le montre: l'idéologie juridique de la «loi»s'accompagne de l'occultation de la dimension concrète des choses auxquelles la norme légale «s'applique», occultation qui caractérise (ou caractérisait généralement jusqu'à certaines réélaborations actuelles) le concept des sciences exactes.

2.3. L'importation en théorie du droit de concepts pris dans l'herméneutique peut aussi servir d'illustration. Souvent, les théoriciens de cette approche ont cité l'interprétation juridique comme modèle - l'interprétation pratiquée par les juristes, et non pas celle de la théorie positiviste de la loi. Mais l'origine de la théorie est ailleurs: dans l'interprétation des textes sacrés et des textes littéraires. Le concept central est celui de «texte» (comme celui de «loi » dans les sciences).

Lexis, cit 
Mais il ne se comprend que dans la relation qu'il implique entre auteur et lecteur, telle qu'elle est objectivée dans le texte, qui la médiatise et porte avec lui toutes les lectures qui en ont été faites, comme sa tradition propre: autonomie du texte, dans sa distance par rapport aussi bien à l'auteur qu'au lecteur, dans une pluralité de sens dont seul un archilecteur ${ }^{9}$ pourrait rendre compte.

Le concept de texte n'est rien sans cette théorie de concepts. Pour qu'il puisse être importé, il faut que les situations scientifiques - c'est-à-dire le problème à résoudre dans chacune des deux disciplines - soient d'une structure analogue. Or, elles le sont pour l'exégèse des Saintes Ecritures, pour l'analyse d'un poème, pour l'interprétation d'une loi: un texte dont le sens doit être déterminé alors que son auteur l'a posé comme un ensemble fini de signes destiné à un ensemble indéterminé de personnes. La similitude du problème permet la transposition des concepts. Texte, signes, auteur, lecteur, distance historique, autonomie: tout cela peut être repris de l'herméneutique littéraire ou sacrée, non pas tel quel, nous le verrons, mais recontextualisé.

Tel n'est pas le cas de la théorie de la «loi ». Si on définit la loi comme la possibilité de la détermination exhaustive du concret par l'abstrait, sont impliqués d'autres concepts: notamment une définition du concret comme réductible à des caractéristiques commensurables communes, une définition de l'universel abstrait qui dépasse la singularité historique et la finitude des choses. Or, ces caractéristiques font précisément défaut à l'ordre juridique, et l'importation du concept de «loi » (non du terme!), qui les charrie avec elle, condamnait la théorie du droit à trahir ce qui pourtant est le propre de l'activité juridique.

Il est clair aussi que chacun de ces deux théories véhicule sa propre idéologie (au sens neutre du terme). Celle de la loi: l'opposition entre Etat et société civile composée d'un ensemble de sujets dont l'égalité de droit n'est respectée que si la loi qui les régit les considère comme de purs sujets, c'est-à-dire dans le mode de l'universel abstrait. Celle du texte: l'historicité singulière des situations à partir desquelles un sens peut être formulé, ce qui implique aussi bien la relativité que l'autonomie de la dimension politique concrète. Si je savais seulement quel ensemble de concepts sera sécrété par l'idéologie de la globalisation! Reste qu'ici je ne fais que soupçonner qu'il est en ébauche avec l'image du réseau, dans la pseudo-égalité des lignes et des points qui le constituent.

2.4. Mais revenons à la théorie du texte et à sa transposition. Le concept de texte appartient à la théorie herméneutique; mais il appartient aussi à d'autres théories, où il répond à d'autres questions et est intégré dans d'autres séries. Ainsi, le texte juridique est, dans la théorie du droit (mais non dans la théorie littéraire ou dans la théologie), en relation avec l'institution de la loi (terme pris ici au sens juridique); il est par là à mettre en rapport avec le concept d'aménagement institutionnel de l'Etat que l'on désigne par les termes de séparation des pouvoirs. C'est un autre concept de distance qui est introduit, qu'il faudra différencier de la «distance» herméneutique classique - une distance qui éloigne l'une de l'autre l'adoption du droit et son application. Dès lors, les concepts d'auteur et de lecteur doivent être repositionnés pour être en mesure de rendre compte à la fois de la

9 Jean-Claude Passeron a attiré notre attention sur ce concept de Michaël Riffaterre. 
problématique herméneutique (comment déterminer le sens d'un texte?) et de la problématique politique (comment le droit peut-il légitimer l'exercice du pouvoir?).

Autrement dit: qu'en est-il de l'»auteur» lorsque celui-ci est législateur? et qui est «lecteur», et à quel titre? enfin, quel est le statut de textes qui se veulent être des normes? Que l'»auteur» est une institution, que le «lectorat» (juges, commentateurs, citoyens) est très hétérogène, que la finalité du texte est spécifique: cela conduit nécessairement à une herméneutique spécifique au $\operatorname{droit}^{10}$.

Que peut-on dire alors du concept de texte? «Dès lors qu'il s'agit, non pas d'additionner des connaissances, mais de produire des savoirs articulant explicitement des concepts issus de disciplines différentes, la question centrale devient celle de la transférabilité des concepts entre les disciplines», écrit Denise Pumain ${ }^{11}$. Et elle continue: «Il s'agit de déconstruire des approches disciplinaires en vue de reconstruire un autre savoir à un niveau plus englobant». Mais que fautil comprendre comme étant un niveau plus englobant? Serait-ce un superconcept de texte, qui serait le genre texte et se subdiviserait par différences spécifiques juridique, littéraire, etc.? On suivrait le modèle de l'arbre de Porphyre, dont on prétend cependant qu'il est dépassé, et à juste titre: notre exemple le montre, puisque l'espèce herméneutique juridique devrait aussi être située en même temps sur un autre arbre, celui de la philosophie du droit (et, par là, de l'Etat).

2.5. La première méthode préconisée par Denise Pumain ${ }^{12}$ pour accéder à un niveau plus englobant introduit deux notions que je n'arrive pas à concrétiser avec le cas du texte, et sur lesquelles elle pourrait apporter quelques commentaires explicatifs. Qu'est-ce qu'une «question d'importance générale»? Comment la formuler dans sa généralité? En l'occurrence - c'est-à-dire quant au concept de «texte»-, on pourrait peut-être la poser ainsi: comment déterminer le sens d'une déclaration quelconque? Arrive-t-on par ce chemin à une « réponse d'importance générale» qui irait plus loin qu'une structure purement formelle - comme l'est sans doute ici la trilogie «auteur - texte - lecteur»? - laquelle n'est peut-être rien d'autre qu'une définition triviale de ce qu'est une déclaration?

La seconde notion est celle de «valeur commune» que présenteraient des «acquis marqués par une approche disciplinaire» «pour l'ensemble des sciences sociales», acquis qui «seraient alors inscrits dans l'état provisoire des connaissances cumulables ». Il est clair que l'approche herméneutique présente un acquis (un ensemble de concepts) qui peut être utilisé pour l'approche de la théorie juridique. Cet acquis, cependant, est-il autre chose qu'une série de concepts permettant de formuler autrement une problématique proprement juridique, et dont l'origine extérieure pourrait parfaitement être oubliée, comme si ces concepts n'avaient servi que de catalyseur à un renouvellement de la théorie du droit?

Ou bien ces «acquis» - en tant que réponse à une «question d'importance générale»-seraient-ils intégrés dans une théorie - une «longue suite»? On aurait par exemple une herméneutique générale et des herméneutiques spéciales, dont il faudrait savoir si elles pourraient constituer des modulations de la générale ou se

\footnotetext{
1o Nous nous permettons de renvoyer à l'ouvrage déjà cité.

11 Dans son texte, ci-dessus.

12 Ibidem.
} 
définiraient plutôt par les dérogations qu'elles présenteraient - les «fausses notes » par rapport à la suite modèle.

Ce sont là des questions que je n'ai pas résolues, bien que j'aie tenté d'appliquer le modèle herméneutique aux textes juridiques; cela m'a permis d'arriver à des conclusions de philosophie du droit. D'où un soupçon: ce point d'arrivée - la philosophie - n'est-il pas le seul qui soit atteignable?

2.6. J'aurais tout autant besoin d'explications complémentaires pour la seconde méthode suggérée par Denise Pumain ${ }^{13}$. J'éprouve déjà une incertitude quant au concept d' «objet commun». La même réalité est-elle un objet commun lorsqu'elle est appréhendée par plusieurs disciplines? On peut faire une analyse littéraire du Code Napoléon, dans la lecture duquel Stendhal se plongeait pour y épurer son style. En quoi ce texte peut-il être considéré comme un objet commun aux approches historiques, stylistiques, idéologiques, juridiques? Denise Pumain évoque certains échecs d'une telle multidisciplinarité polarisée par l'objet, et aimerait que le rapprochement des points de vue et des méthodes communs se situe «plus en amont». Elle semble cependant illustrer son propos en citant des contre-exemples dans lesquels des économistes auraient repris des théories sociales ou géographiques dépassées dans leur propre discipline.

Il y a, dirais-je, un premier problème, qui est de temps : non seulement le temps dont on dispose pour accéder à d'autres disciplines que la sienne, mais aussi le temps des disciplines elles-mêmes, dont, de l'extérieur, on apprend quelquefois les innovations seulement lorsque, banalisées, elles sont dépassées. Ainsi beaucoup de juristes peinent avec la philosophie post-kantienne (c'est dire s'ils ont pris du retard!), mais, inversement, beaucoup de philosophes n'ont guère de familiarité avec le droit en tant que pratique.

C'est sans doute ce défaut de familiarité avec la discipline étrangère qui constitue le handicap le plus lourd. Et je définirais la familiarité, non pas par la connaissance des acquis et des résultats, mais par celle des problématiques: quel est le positionnement des questions que la discipline cherche à résoudre, quel est l'appareil conceptuel dans lequel ces questions peuvent être simplement posées. Peut-être l'analyse des problématiques permettrait plus que celle des acquis de situer un objet commun, par les questions qui lui sont posées et la diversité des intérêts qui provoquent ces questions. Mais on retrouverait alors la philosophie.

\section{Faculté de Droit, Université de Lausanne}

13 Ibidem. 